\title{
On the Performance of the Time Reversal SM-MIMO-UWB System on Correlated Channels
}

\author{
Hieu Nguyen, ${ }^{1}$ Van Duc Nguyen, ${ }^{2}$ Trung Kien Nguyen, ${ }^{1}$ Kiattisak Maichalernnukul, ${ }^{1}$ \\ Feng Zheng, ${ }^{3}$ and Thomas Kaiser ${ }^{3}$ \\ ${ }^{1}$ Institute of Communications Technology, Leibniz University of Hannover, 30167 Hannover, Germany \\ ${ }^{2}$ School of Electronics and Telecommunications, Hanoi University of Science and Technology, 1 Dai Co Viet, Hanoi 10000, Vietnam \\ ${ }^{3}$ Institute of Digital Signal Processing, Faculty of Engineering, University of Duisburg-Essen, 47057 Duisburg, Germany
}

Correspondence should be addressed to Hieu Nguyen, hieunth@gmail.com

Received 3 January 2012; Revised 9 March 2012; Accepted 9 March 2012

Academic Editor: David A. Sanchez-Hernandez

Copyright (c) 2012 Hieu Nguyen et al. This is an open access article distributed under the Creative Commons Attribution License, which permits unrestricted use, distribution, and reproduction in any medium, provided the original work is properly cited.

\begin{abstract}
The impact of spatial correlation on the multi-input multi-output ultrawide band (MIMO-UWB) system using the time reversal (TR) technique is investigated. Thanks to TR, several data streams can be transmitted by using only one antenna in a system named virtual MIMO-TRUWB. Since the virtual MIMO-TR-UWB system is not affected by the transmit correlation, under the condition of the high spatial correlation, it outperforms the true MIMO-UWB system with multiple transmit antennas. The channel measurements are performed in short-range indoor environment, both line-of-sight and non-line-of-sight to verify the adopted correlated channel model.
\end{abstract}

\section{Introduction}

An ultrawide band (UWB) communication system, whose relative bandwidth is usually defined as greater than twentyfive percents, has become a promising candidate for high data rate and short-range communication systems, which have attracted great interests from both academic and industrial aspects recently $[1,2]$. Impulse radio UWB is however designed with low complexity and low power consumption applications such as in many application such as wireless sensor networks, sensing and positioning systems, interchip communication, contact less wireless, biological or biomedical networks, imaging systems, health monitoring, and body-area networks [3]. However, due to the wide bandwidth property, UWB systems suffer from a very long delay spread by multipath effect [4-6]. One has to deploy RAKE receivers to combat the intersymbol interference (ISI).

The time reversal (TR) technique, which is originated from under-water acoustics and ultrasonic [7, 8], now has been used in many applications such as localization, imaging and green wireless communications [9-14]. TR also has shown its potential in dealing with the ISI problems in UWB $[15,16]$. In a TR system, the time-reversed channel impulse response (CIR) is implemented as a filter at the transmitter side. This process leads to a very narrow focus of power at the receiver at one specific time instant, and one specific space position if the CIRs between any two communication pairs at different locations are de-correlated. In other words, a TRUWB prefiltering system has a unique feature of space-time domain focusing.

The space-time focusing feature is also beneficial in a MIMO spatial multiplexing scheme $[17,18]$. Several studies have applied the TR technique to multiple antennas beamforming systems. In [19-21], the same stream of data is beamformed by a TR filter and transmitted over transmit antennas. In [22, 23], a joint ZF and TR preequalizer is designed to minimize the ISI and maximize the received power at the intended receiver. This proposal only deals with the ISI problem for beamforming schemes. The potential of a MIMO UWB system using spatial multiplexing scheme is considered in [24], where the matching filter plays the role of a passive time reversal filter and the maximum likelihood (ML) detector is used to deal with the multistream interference (MSI) but ignores the ISI. The SM-MIMO-UWB system using TR is introduced in [25] with the capability of transmit 
antenna selection. The TR technique and preequalizer are proposed in [26] for the so-called virtual MIMO UWB system.

As shown in [26], with the help of the TR prefilter and a properly designed preequalizer, the system with only one transmit antenna can deliver several independent data streams at the same time. However, the spatial correlation among the transmit and receive antennas has not been investigated. We have taken the spatial correlation into account in [27], where a constant spatial correlation model for MIMO UWB with linear array structure has been applied. In this paper, the performance of system over both correlated lineof-sight (LOS) and non-line-of-sight (NLOS) channels is investigated with the same correlation model. In order to verify the adopted correlation model, the correlated channels are measured in both scenarios NLOS and LOS in indoor environment. These scenarios are referred to as channel models CM1 and CM2, respectively, in the IEEE 802.15.3a standard [28]. The BER results on the adopted correlated channel model with an appropriate value of correlation coefficient are shown closely matching with those on the measured indoor channel.

It is well known that the MIMO-TR-UWB system can achieve transmit diversity [26], but it suffers from penalty caused by both transmit and receive antenna correlations. Meanwhile, the single-input multiple-output TRUWB (SIMO-TR-UWB) or virtual MIMO-TR-UWB does not face the transmit antenna correlation because it has only one transmit antenna. It will be shown that, under some conditions, the virtual MIMO outperforms the true MIMO system in term of the BER performance.

The remainder of the paper is organized as follows: in Section 2, the correlated MIMO channel model is shown, and then the virtual MIMO-UWB-TR system is presented in Section 3; UWB channel measurement is described in Section 4; in Sections 5 and 6, numerical simulation results and conclusions are presented, respectively.

\section{Correlated MIMO Channel Model}

In order to achieve the high data rate without expanding the bandwidth, the spatial multiplexing scheme with multiple transmit antennas is introduced. In the spatial multiplexing (SM) system, several streams of data are transmitted over several transmit antennas at the same time. The channel capacity can be increased proportionally to the number of antennas. Let us consider an SM-MIMO UWB system with $M$ transmit and $N$ receive antennas as shown in Figure 1, where the preequalizer and prefilter blocks will be discussed in the next section. We assume that the maximum length of each channel realization is $L$. The CIR between transmit antenna $j$ and receive antenna $i$ is

$$
h_{i, j}(t)=\sum_{l=1}^{L} \alpha_{l}^{i, j} \delta\left(t-\tau_{l}^{i, j}\right), \quad i=1, \ldots, N, j=1, \ldots, M .
$$

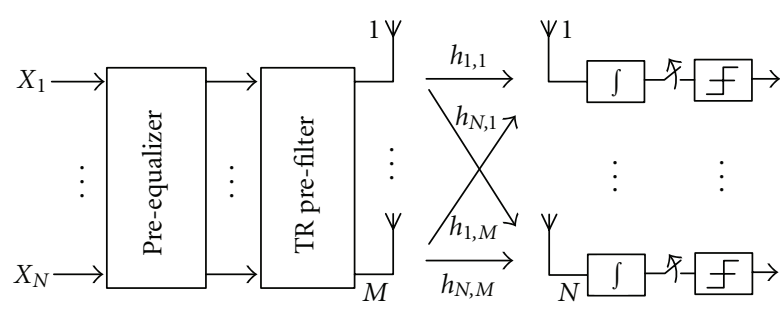

Figure 1: Block diagram of an SM-MIMO UWB system.

We can arrange these channels in a matrix form as follows:

$$
\mathbf{H}(t)=\left(\begin{array}{cccc}
h_{1,1}(t) & h_{1,2}(t) & \cdots & h_{1, M}(t) \\
h_{2,1}(t) & h_{2,2}(t) & \cdots & h_{2, M}(t) \\
\vdots & \vdots & \ddots & \vdots \\
h_{N, 1}(t) & h_{N, 2}(t) & \cdots & h_{N, M}(t)
\end{array}\right) .
$$

Generally, the entries of the MIMO channel matrix $\mathbf{H}$ are assumed to be independent of each other. In the real world, however, the spatial correlation among antennas (transmit or receive antennas) exists. In other words, the individual channels of $\mathbf{H}$ are correlated $[29,30]$. The correlation is caused by a variety of reasons such as inadequate antenna spacing.

The correlation can be included into the MIMO UWB channel model by introducing fixed transmit and receive correlation matrices following the well-known Kronecker model. The transmit and receive correlation can be represented by an $M \times M$ matrix $\mathbf{R}_{T x}$ and an $N \times N$ matrix $\mathbf{R}_{R x}$, respectively [31]. The correlated channel is represented by equation

$$
\mathbb{H}=\mathbf{R}_{R x}^{1 / 2} \mathbf{H}_{w} \mathbf{R}_{T x}^{1 / 2},
$$

where $\mathbf{H}_{w}$ is the channel matrix of independent channel realization.

For the fixed correlation matrix in the Kronecker model, there are some variations in terms of whether or not the impact of interelement distance is considered [32, 33]. In this paper, the exponential decay model for the correlation is deployed [30,32]. The transmit and receive correlation matrices are

$$
\begin{aligned}
\mathbf{R}_{T x} & =\left(\begin{array}{ccccc}
1 & \rho_{T x} & \rho_{T x}^{2} & \cdots & \rho_{T x}^{M-1} \\
\rho_{T x} & 1 & \rho_{T x} & \cdots & \rho_{T x}^{M-2} \\
\vdots & \vdots & \vdots & \ddots & \vdots \\
\rho_{T x}^{M-1} & \rho_{T x}^{M-2} & \rho_{T x}^{M-3} & \cdots & 1
\end{array}\right), \\
\mathbf{R}_{R x} & =\left(\begin{array}{ccccc}
1 & \rho_{R x} & \rho_{R x}^{2} & \cdots & \rho_{R x}^{N-1} \\
\rho_{R x} & 1 & \rho_{R x} & \cdots & \rho_{R x}^{N-2} \\
\vdots & \vdots & \vdots & \ddots & \vdots \\
\rho_{R x}^{N-1} & \rho_{R x}^{N-2} & \rho_{R x}^{N-3} & \cdots & 1
\end{array}\right),
\end{aligned}
$$

where $\rho_{T x}$ and $\rho_{R x}$ are the correlation coefficients of transmit and receive antennas, respectively. 


\section{Virtual MIMO-UWB-TR System}

In this paper, a TR prefilter combined with a zero forcing (ZF) preequalizer, which has been proposed in $[26,27]$, is adopted to combat the ISI and the MSI in the SM-MIMO UWB system. [27]

The TR prefilter matrix of the MIMO system is given by

$$
\overline{\mathbf{H}}(t)=\left(\begin{array}{cccc}
h_{1,1}(-t) & h_{2,1}(-t) & \cdots & h_{N, 1}(-t) \\
h_{1,2}(-t) & h_{2,2}(-t) & \cdots & h_{N, 2}(-t) \\
\vdots & \vdots & \ddots & \vdots \\
h_{1, M}(-t) & h_{2, M}(-t) & \cdots & h_{N, M}(-t)
\end{array}\right)
$$

which is based on the original CIR matrix reversed in time and transposed in space. The CIR matrix of the equivalent channel is

$$
\hat{\mathbf{H}}(t)=\left(\begin{array}{cccc}
\hat{h}_{1,1}(t) & \hat{h}_{1,2}(t) & \cdots & \hat{h}_{1, N}(t) \\
\hat{h}_{2,1}(t) & \hat{h}_{2,2}(t) & \cdots & \hat{h}_{2, N}(t) \\
\vdots & \vdots & \ddots & \vdots \\
\hat{h}_{N, 1}(t) & \hat{h}_{N, 2}(t) & \cdots & \hat{h}_{N, N}(t)
\end{array}\right),
$$

where each component of the equivalent composite channel (which we will call equivalent channel in the sequel for simplicity) is calculated by

$$
\hat{h}_{i, j}(t)=\sum_{k=1}^{M} h_{i, k}(t) \otimes h_{j, k}(-t), \quad i, j=1, \ldots, N .
$$

Some remarks can be drawn from the matrix of the equivalent channel. Firstly, the maximum number of independent data streams the system can achieve is $N$, which is the number of receive instead of transmit antennas. Secondly, the matrix of the equivalent channel is a square matrix with the entries in the main diagonal being the summation of the autocorrelation of the original CIRs and other entries being the summation of the cross-correlation of the original CIRs between the transmit and receive antennas. Third, the TR technique in MIMO-UWB can exploit $M$ order transmit diversity, and the diversity gain depends on the number of transmit antennas.

Let us consider the special case of MIMO-TR-UWB system, where the data is transmitted over only one antenna, that is, $M=1$. The channel matrix is thus only a column vector $\mathbf{H}(t)=\left[h_{1}(t), h_{2}(t), \ldots, h_{N}(t)\right]^{T}$ and the TR filter matrix is a row vector $\overline{\mathbf{H}}(t)=\left[h_{1}(-t), h_{2}(-t), \ldots, h_{N}(-t)\right]$. The equivalent channel, however, is still a square $N \times N$ matrix as presented in (6). Each entry of equivalent matrix is

$$
\hat{h}_{i, j}(t)=h_{i}(t) \otimes h_{j}(-t), \quad i, j=1, \ldots, N .
$$

In this case, $N$ data stream are multiplexed to be transmitted over one antenna. In other words, the data are seen to be transmitted over $N$ virtual antennas. Hence, the system is named virtual MIMO-TR-UWB.
In the true MIMO-TR-UWB system, when the separation distance between antenna elements is small, the spatial correlation appears at both transmit and receive sides. These correlation will doubly degrade the performance of the system. Meanwhile, in the virtual MIMO-TR-UWB system, the correlation appears only at the receiver side and the degradation is caused only by receiver correlation. The virtual MIMO-TR-UWB system can mitigate the impact of the transmit correlation. However, it cannot achieve the transmit diversity gain as the true one does.

The CIR can also be reused by the preequalizer design at the transmitter side for further multistream interference (MSI) mitigation. Here, we assume that a ZF preequalizer is deployed. A simple TR filter focuses the energy into a short-time duration in the equivalent CIR, so we can use the shortened equivalent channels to design the preequalizer. Suppose that the maximum length of the equivalent channel is $L_{e}$. As shown in [27], we can choose $L_{s}\left(L_{s} \ll L_{e}\right)$ capturing most of the energy to compute the linear preequalizer.

We assume that the channels do not change when a block of $K+L_{s}-1$ data symbols is transmitted. The new equivalent channel matrix can be represented by a block Toeplitz matrix

$\hat{\mathbf{H}}_{s}=\left(\begin{array}{cccccc}\hat{\mathbf{H}}\left[L_{s}-1\right] & \cdots & \hat{\mathbf{H}}[0] & 0 & \cdots & 0 \\ 0 & \hat{\mathbf{H}}\left[L_{s}-1\right] & \cdots & \hat{\mathbf{H}}[0] & \cdots & 0 \\ \vdots & \ddots & \ddots & \ddots & \ddots & \vdots \\ 0 & \cdots & 0 & \hat{\mathbf{H}}\left[L_{s}-1\right] & \cdots & \hat{\mathbf{H}}[0]\end{array}\right)$,

where each block matrix is

$$
\hat{\mathbf{H}}[k]=\left(\begin{array}{cccc}
\hat{h}_{1,1}[k] & \hat{h}_{1,2}[k] & \cdots & \hat{h}_{1, N}[k] \\
\hat{h}_{2,1}[k] & \hat{h}_{2,2}[k] & \cdots & \hat{h}_{2, N}[k] \\
\vdots & \vdots & \ddots & \vdots \\
\hat{h}_{N, 1}[k] & \hat{h}_{N, 2}[k] & \cdots & \hat{h}_{N, N}[k]
\end{array}\right) .
$$

The preequalizer matrix $\mathbf{G}_{Z F}$ is the inverse of the shortened equivalent channels matrix, given by $[27,34]$

$$
\mathbf{G}_{Z F}=\alpha \hat{\mathbf{H}}_{s}^{\dagger}=\alpha\left(\hat{\mathbf{H}}_{s}^{H} \hat{\mathbf{H}}_{s}\right)^{-1} \hat{\mathbf{H}}_{s}^{H},
$$

where $t$ denotes the Moore-Penrose pseudoinverse of a matrix. The coefficient $\alpha$, which can be found from [34], is introduced for the power constraint of the transmit signal.

\section{UWB Channel Measurement}

We generate the channel realizations for comparison using both the adopted correlated channel model and those obtained from our indoor measurements. The indoor UWB channels are measured in time domain within a $4.7 \mathrm{~m} \times$ $6.3 \mathrm{~m}$ room with a table, a chair, a wooden rack, and different kinds of smaller scattering objects as shown in Figure 2(a). The floor and walls of this room are constructed from concrete materials. The ceiling ( $3.2 \mathrm{~m}$ above the floor) is composed of iron sheets and metallic beams. 


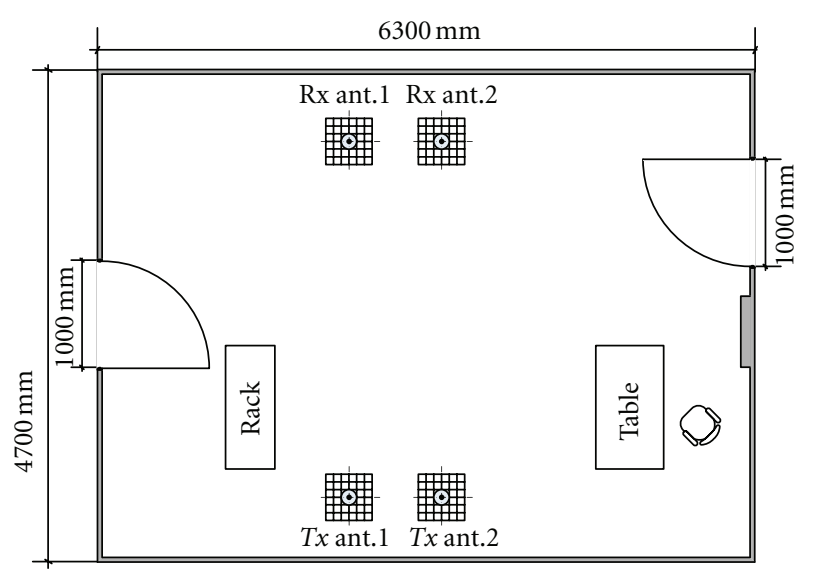

(a) Floor plan and measurement configuration

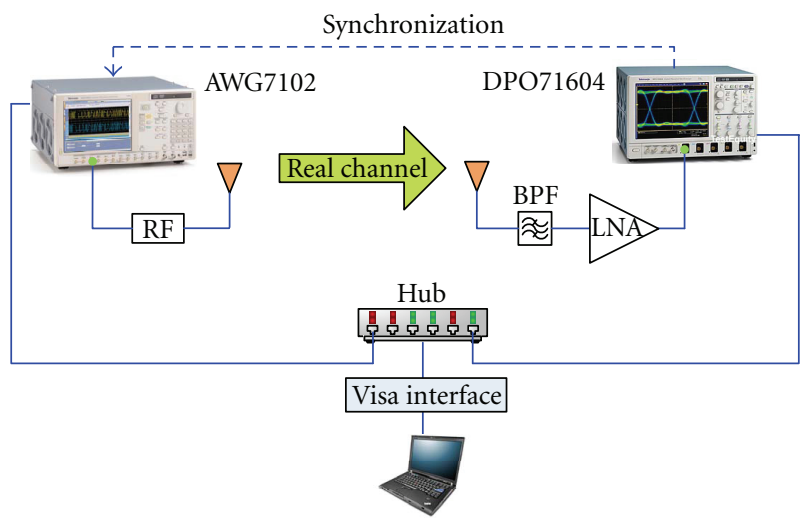

(b) A sketch of system

Figure 2: Setup of UWB channel measurement.

The sketch of the whole system is illustrated in Figure 2(b). The major components used in the measurements are a Tektronix AWG7102 arbitrary waveform generator (AWG) and a Tektronix DPO71604 digital phosphor oscilloscope (DPO) which are synchronized by the reference clock signal and connected to the processing computer via Ethernet. The AWG supports two channels with sampling rates of up to $10 \mathrm{GSamples} / \mathrm{s}$ (GS/s) and $3.5 \mathrm{GHz}$ bandwidth or one channel with sampling rates of up to $20 \mathrm{GS} / \mathrm{s}$ and $5.8 \mathrm{GHz}$ analog bandwidth using interleaving. The DPO provides four channels with sampling rates of up to $50 \mathrm{GS} / \mathrm{s}$ and $16 \mathrm{GHz}$ frequency span. The small-size omnidirectional UWB patch antennas are used. On the receiver side, each antenna is connected to the DPO through a bank of bandpass filters ( $1.5 \mathrm{GHz}$ bandwidth) and $+55 \mathrm{~dB}$ low-noise amplifiers for reliable signal acquisition under harsh SNR conditions. The transmit and receive antennas are placed at the same height of $1.1 \mathrm{~m}$. The probe UWB pulse used in all measurements is a second-order derivative Gaussian pulse with the symbol duration $T=250 \mathrm{~ns}$.

The common scenario for applications of UWB systems is in a small office or home environment. Therefore, we focus on the short-range indoor environment, both NLOS and LOS. These scenarios are referred to as CM1 (for LOS)

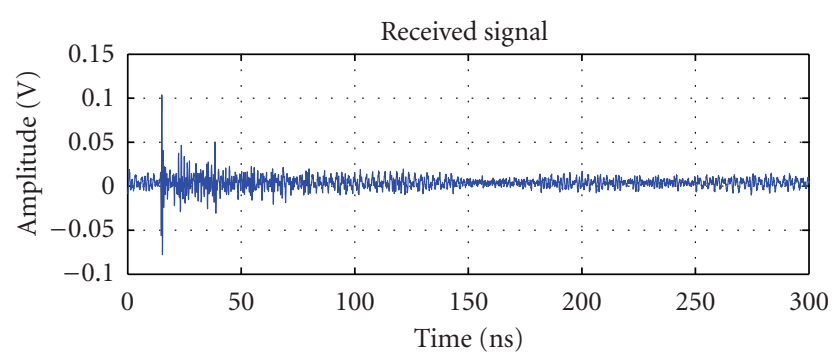

(a)

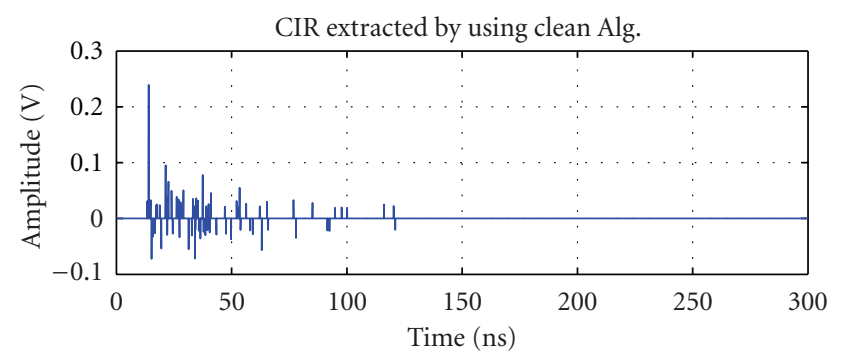

(b)

FIGURE 3: An example of the received signal and the extracted CIR.

and CM2 (for NLOS) in the IEEE 802.15.3a UWB channel models [28]. In our measurement, the blockage for the NLOS scenario is created by vertically placing a large iron sheet in the direct propagation path.

The temporal stationarity of the environment is ensured by the absence of mobile objects/persons, thus allowing us to assume that the channels for the links are quasistatic over the duration of data transmissions. For the MIMO channels, the spatial antenna arrays at both transmitter and receiver are synthesized by sequential measurements exploiting such temporal stationarity. Due to this array synthesis, our measurement results do not include the effects of antenna coupling. A $(2 \times 2)$ virtual MIMO channel, corresponding to 4 SISO channels ( 49 realizations for each), are measured in our campaign. The adjacent antennas are separated by $0.2 \mathrm{~m}$, and the mean separation between transmit and receive antennas is kept at $4 \mathrm{~m}$.

In order to extract the CIR from received signals, we use the well-known deconvolution technique, the so-called Clean Algorithm [35]. A realization of the SISO channel which is extracted from the received signal by using Clean Algorithm is shown in Figure 3.

\section{Numerical Results and Discussions}

In our simulations, the binary data is modulated to the binary phase-amplitude modulation format. The UWB monocycle waveform is the 2nd derivative Gaussian pulse, represented by

$$
p(t)=\left[1-4 \pi\left(\frac{t-t_{c}}{w}\right)^{2}\right] e^{-2 \pi\left(\left(t-t_{c}\right) / w\right)^{2}},
$$

where $w$ is a parameter corresponding to pulse, width, $t_{c}$ is a time shifting of the pulse and $T$ is the symbol duration. In our simulations, $w=1 \mathrm{~ns}, t_{c}=w / 2$, and $T=2 \mathrm{~ns}$. 
Simulations results are based on the UWB channel model in the IEEE 802.15.3a standard [28] and those obtained from measurements. Results are followed by descriptions of the channel models. The correlated MIMO channel model is generated by modifying the channel models in the IEEE 802.15.3a standard, which is formulated for SISO UWB, then included with the correlation matrices in (5). For the simulation results based on the standard model, the correlation of receive antennas affects in the same way on SIMO and MIMO-TR-UWB systems and the receive correlation coefficient is fixed at $\rho_{R x}=0.2$. For the fair comparison, we shorten the equivalent channel when designing the ZF preequalizer with the same ratio $L_{s} / L_{e}=0.2$ for standard channels and measured channels.

Figure 4 shows the BER performance of a $2 \times 2 \mathrm{MIMO}$ system operating in the modified CM1, CM2 channels with the matched transmit correlation coefficient and in the measured LOS and NLOS channels. The value of correlation coefficient matched with the measurement result is found by exhaustive search and is then rounded to one decimal place. It can be seen that the average BERs based on realizations of CM1 and CM2 with the selected value of $\rho_{T x}=0.2$ are closely matched with those based on the corresponsive measured channels. It is noted that this correlation value is specific to our measurement setting. Also observed from this figure that the system performance in the LOS environment is better than in the NLOS environment for standard channels as well as measured channels. Transmission through walls and obstructions in NLOS environment leads to additional delay before arriving at the receiver and significant additional attenuation, which reduce the received strength and lead to increased errors in estimation [36].

In Figures 5 and 6, the effect of the transmit correlation coefficient on the BER performance of the system is presented. In both figures, two data streams are transmitted over $M=1,2$, and 4 transmit antennas, respectively. The transmit correlation coefficient $\rho_{T x}$ varies from 0 (no correlation) to 0.95 (strong correlation), and the $\mathrm{SNR}=12 \mathrm{~dB}$.

It can be seen from Figure 5, for the LOS environment, that, when $\rho_{T x}$ increases, the average BER of the SIMO or virtual MIMO-TR-UWB system keeps unchanged while it increases in the true MIMO case. This can be explained by that the SIMO system is impacted by only the spatial correlation between receive antennas, so the BER performance of such system does not change with variation of the transmit correlation. Meanwhile, the spatial correlation appears at both transmit and receive sides in the MIMO system and it will doubly degrade the performance of the system. The BER performance of the MIMO system will be worse than the SIMO system if the transmit correlation coefficient is greater than a critical value. For example, the performance of the 2 $\times 2$ MIMO system is worse than the $1 \times 2$ SIMO system if $\rho_{T x} \geq 0.7$, and the performance of the $4 \times 2$ MIMO system is worse than the $1 \times 2$ SIMO system if $\rho_{T x} \geq 0.6$.

At low transmit correlation regime, the more the transmit antennas, the better the BER performance that the system can achieve. This is the advantage of the transmit diversity possessed by the MIMO-TR-UWB system [34]. However, if the correlation is strong, more transmit antenna

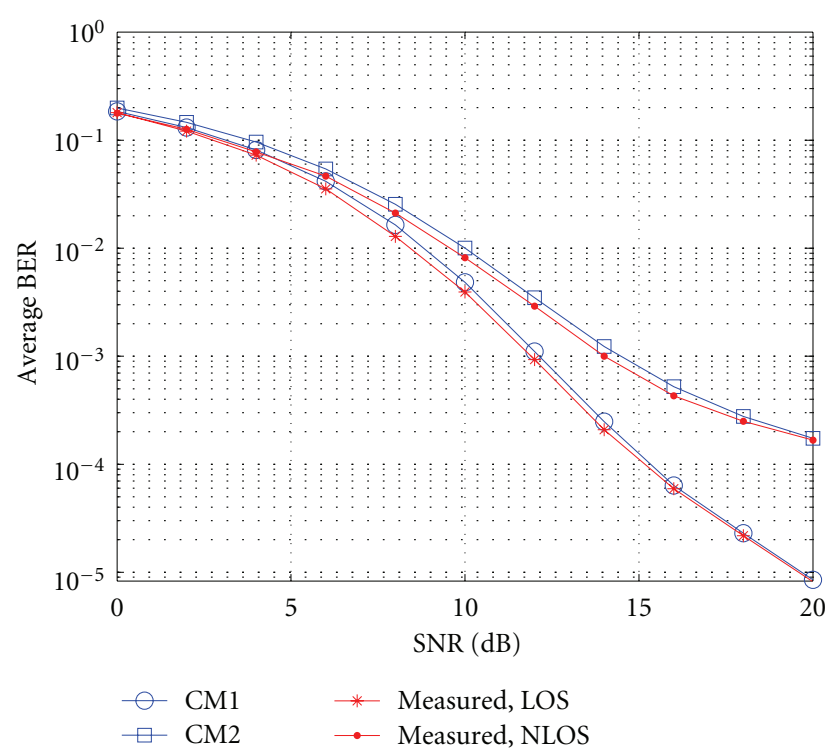

FIGURE 4: BER performance comparison, for standard channels with $\rho_{T x}=0.2$ and for measured indoor channels.

will greatly degrade the performance of MIMO systems since the rank of the MIMO channel reduces, leading to destroy the diversity. As shown in Figure 5, when the transmit correlation coefficient $\rho_{T x}$ is greater than 0.45 , the performance of the $2 \times 2$ MIMO system outperforms the performance of the $4 \times 2$ MIMO system. It has been shown in Figure 5 that, at high correlation, the BER increases from 0.002 to 0.0024 . This trend is also observed in Figure 6 for the NLOS environment. In CM1 channel, BER degradation of MIMO system in comparison with SIMO system is about 0.0005 , while, in CM2 channel, this value is 0.0015 .

The BER performance of $1 \times 2$ SIMO and $2 \times 2$ MIMO systems operating in the CM1 and CM2 with some values of $\rho_{T x}$ is illustrated in Figures 7 and 8. As can be seen from these figures, if there is no correlation, the MIMO-TR-UWB system achieves very good BER performance. However, if the correlation appears, the average BER will reach an error floor at the high SNR. Particularly, the performance of the MIMO system will be worse than the SIMO system if the transmit correlation is too high (e.g., $\rho_{T x}=0.9$ ). In Figure 7, at BER $=$ $10^{-3}$, the SNR of the MISO scheme $3 \mathrm{~dB}$ worse than the MIMO scheme without correlation but $1 \mathrm{~dB}$ better than the high correlation MIMO scheme. In Figure 8, these values are $5 \mathrm{~dB}$ and $3 \mathrm{~dB}$, respectively. This is also agreed with results in Figures 5 and 6.

\section{Conclusion}

We have investigated the impact of the spatial correlation on the performance of the SM-MIMO UWB systems where the TR prefilter and the ZF preequalizer are adopted to mitigate the interferences. It is shown that the SIMO-TR-UWB system can work as a virtual MIMO system when several data streams are transmitted over only one transmit antenna. The SIMO system suffers from only the receive correlation, 


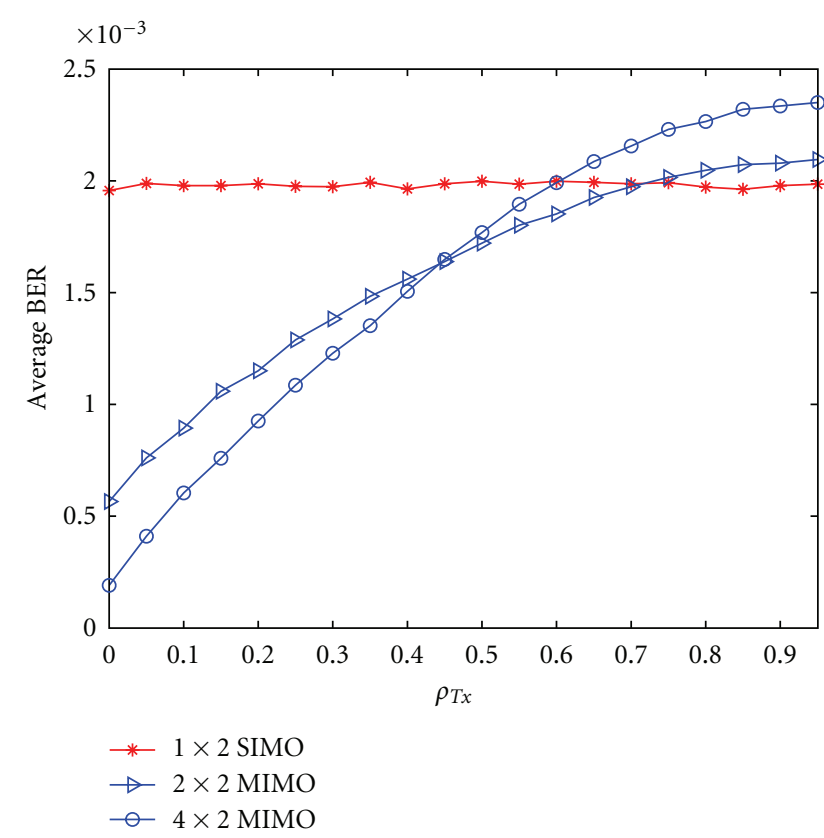

FIGURE 5: BER performance versus transmit correlation coefficient, for CM1 channels.

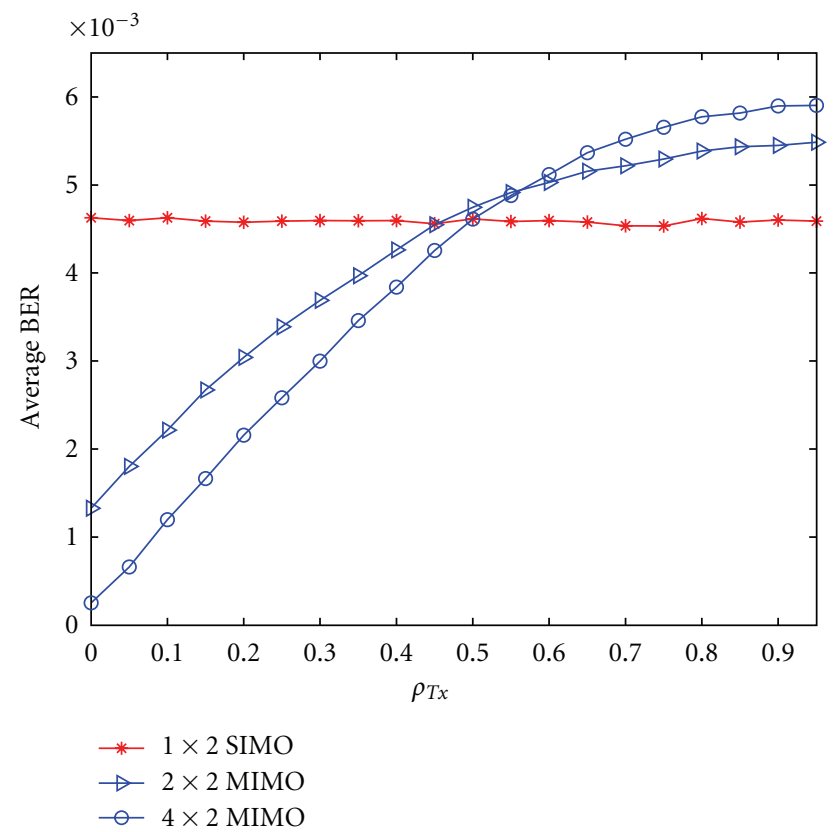

FIGURE 6: BER performance versus transmit correlation coefficient, for CM2 channels.

while both the transmit and receive correlation affect the performance of the real MIMO system. The simulation results show that, at the low transmit correlation regime, the MIMO-TR-UWB systems outperform the SIMO-TRUWB systems in the BER performance. However, when the transmit correlation becomes large, the performance of the MIMO systems is worse than that of the SIMO systems.

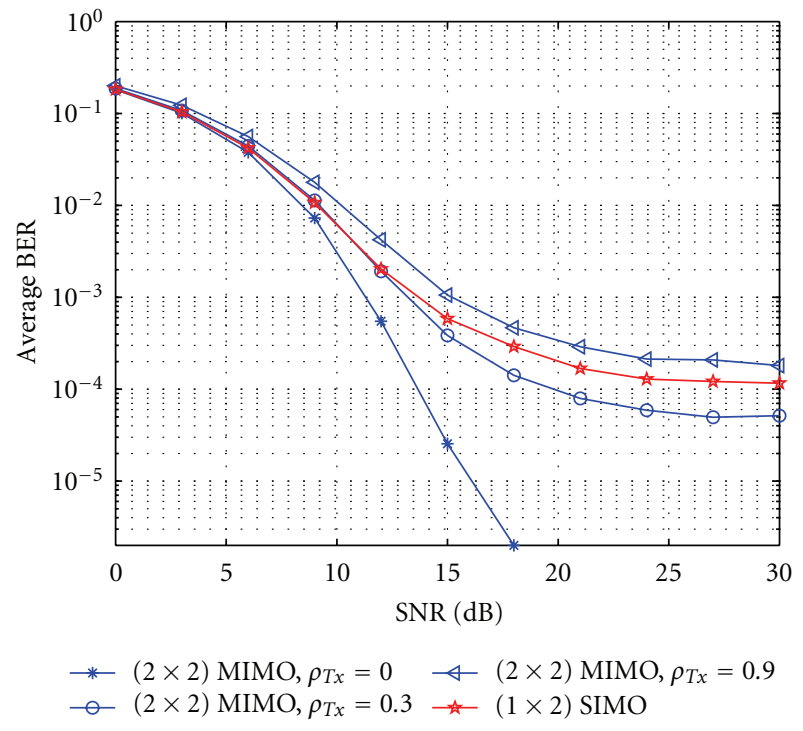

FIGURE 7: BER performance of $2 \times 2 \mathrm{MIMO}$ and $1 \times 2$ SIMO systems, for CM1 channels.

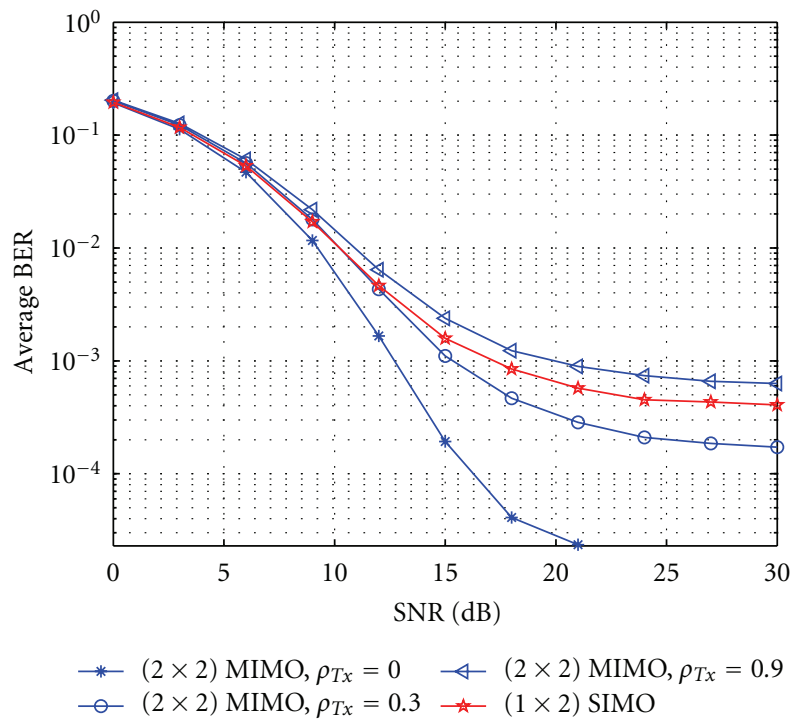

FIGURE 8: BER performance of $2 \times 2$ MIMO and $1 \times 2$ SIMO systems, for CM2 channels.

The BER performance based on the fixed correlation model generated from the standard UWB channel models is compared with those based on the MIMO UWB channels measured in indoor environment and the matched value of the spatial correlation coefficient is found.

Additionally, we would like to point out that it is interesting to combine the design of the TR prefilter and the preequalizer into one-step design since both designs use the same information. It is not clear yet whether or not the integrate design can reduce the complexity in the system 
implementation. However, how to exploit the nice property of the composite TR channel remains an issue in the integrate design.

\section{Acknowledgment}

This paper is carried out under the framework of the Project number 102.02.07.09 funded by the Vietnamese National Foundation for Science and Technology Development (NAFOSTED).

\section{References}

[1] M. Di Benedetto, T. Kaiser, D. Porcino, A. Molisch, and I. Opperman, UWB Communication Systems A Comprehensive Overview, Hindawi Publishing Corporation, New York, NY, USA, 2006.

[2] R. J. Fontana, "Recent system applications of short-pulse ultra-wideband (UWB) technology," IEEE Transactions on Microwave Theory and Techniques, vol. 52, no. 9, pp. 2087 2104, 2004.

[3] J. R. Fernandes and D. Wentzloff, "Recent advances in IRUWB transceivers: an overview," in Proceedings of the IEEE International Symposium on Circuits and Systems: Nano-Bio Circuit Fabrics and Systems (ISCAS '10), pp. 3284-3287, June 2010.

[4] R. J. M. Cramer, R. A. Scholtz, and M. Z. Win, "Evaluation of an ultra-wide-band propagation channel," IEEE Transactions on Antennas and Propagation, vol. 50, no. 5, pp. 561-570, 2002.

[5] A. F. Molisch, K. Balakrishnan, D. Cassioli et al., "IEEE 802.15.4a Channel model final report," Tech. Rep., 2005, http://www.ieee802.org/15/pub/04/15-04-0662-02-004achannel-model-final-report-r1.pdf.

[6] F. Zheng and T. Kaiser, "On the evaluation of channel capacity of multi-antenna UWB indoor wireless systems," in Proceedings of the 8th IEEE International Symposium on Spread Spectrum Techniques and Applications (ISSSTA '04), pp. 525529, September 2004.

[7] M. Fink, "Time reversal of ultrasonic fields. I: basic principles," IEEE Transactions on Ultrasonics, Ferroelectrics, and Frequency Control, vol. 39, no. 5, pp. 555-566, 1992.

[8] A. Derode, P. Roux, and M. Fink, "Robust acoustic time reversal with high-order multiple scattering," Physical Review Letters, vol. 75, no. 23, pp. 4206-4209, 1995.

[9] F. Foroozan and A. Asif, "Time reversal based active array source localization," IEEE Transactions on Signal Processing, vol. 59, no. 6, pp. 2655-2668, 2011.

[10] M. H. S. Sajjadieh and A. Asif, "Unsupervised time reversal based microwave imaging for breast cancer detection," in Proceedings of the 24th Canadian Conference Electrical and Computer Engineering (CCECE'11), pp. 411-415, 2011.

[11] B. Wang, Y. Wu, F. Han, Y.-H. Yang, and K. J. R. Liu, "Green wireless communications: a time-reversal paradigm," IEEE Journal on Selected Areas in Communications, vol. 29, no. 8, pp. 1698-1710, 2011.

[12] M. Rice and M. Saquib, "MIMO equalization for helicopterto-ground communications," in Proceedings of the Military Communications Conference (MILCOM '11), pp. 501-506, 2011.

[13] T. Shimura, H. Ochi, Y. Watanabe, and T. Hattori, "Timereversal communication in deep ocean Results of recent experiments," in Proceedings of the IEEE Symposium on Underwater
Technology (UT) and Workshop on Scientific Use of Submarine Cables and Related Technologies (SSC'11), April 2011.

[14] B. Van Damme, K. Van Den Abeele, and O. Bou Matar, "The vibration dipole: a time reversed acoustics scheme for the experimental localisation of surface breaking cracks," Applied Physics Letters, vol. 100, no. 8, Article ID 084103, 3 pages, 2012.

[15] T. Strohmer, M. Emami, J. Hansen, G. Papanicolaou, and A. J. Paulraj, "Application of time-reversal with MMSE equalizer to UWB communications," in Proceedings of the IEEE Global Telecommunications Conference (GLOBECOM '04), vol. 5, pp. 3123-3127, December 2004.

[16] M. Emami, M. Vu, J. Hansen, A. J. Paulraj, and G. Papanicolaou, "Matched filtering with rate back-off for low complexity communications in very large delay spread channels," in Conference Record of the 38th Asilomar Conference on Signals, Systems and Computers, vol. 1, pp. 218-222, November 2004.

[17] G. J. Foschini, "Layered space-time architecture for wireless communication in a fading environment when using multielement antennas," Bell Labs Technical Journal, vol. 1, no. 2, pp. 41-59, 1996.

[18] J. H. Winters, "On the capacity of radio communication systems with diversity in a Rayleigh fading environment," IEEE Journal on Selected Areas in Communications, vol. 5, no. 5, pp. 871-878, 1987.

[19] P. Kyritsi, G. Papanicolaou, P. Eggers, and A. Oprea, "IMISO time reversal and delay-spread compression for FWA channels at $5 \mathrm{GHz}$," IEEE Antennas and Wireless Propagation Letters, vol. 3, no. 1, pp. 96-99, 2004.

[20] R. C. Qiu, C. Zhou, N. Guo, and J. Q. Zhang, “Time reversal with MISO for ultrawideband communications: experimental results," IEEE Antennas and Wireless Propagation Letters, vol. 5, no. 1, pp. 269-273, 2006.

[21] R. C. Qiu, "A theory of time-reversed impulse MultipleInput Multiple-Output (MIMO) for Ultra-Wideband (UWB) communications," in Proceedings of the IEEE International Conference on Ultra-Wideband (ICUWB '06), pp. 587-592, September 2006.

[22] P. Kyritsi, G. Papanicolaou, and C. Tsogka, "Optimally designed time reversal and zero forcing schemes," in Proceedings of the International Symposium on Wireless Personal Multimedia Communications (WPMC '05), pp. 105-109, September 2005.

[23] P. Kyritsi, P. Stoica, G. Papanicolaou, P. Eggers, and A. Oprea, "Time reversal and zero-forcing equalization for fixed wireless access channels," in Proceedings of the 39th Asilomar Conference on Signals, Systems and Computers, pp. 1297-1301, November 2005.

[24] M. Weisenhorn and W. Hirt, "Performance of binary antipodal signaling over the indoor UWB MIMO channel," in Proceedings of the International Conference on Communications (ICC '03), vol. 4, pp. 2872-2878, May 2003.

[25] H. Nguyen, F. Zheng, and T. Kaiser, "Antenna selection for time reversal MIMO UWB systems," in Proceedings of the 69th IEEE Vehicular Technology Conference (VTC '09), April 2009.

[26] H. Nguyen, Z. Zhao, F. Zheng, and T. Kaiser, "On the MSI mitigation for MIMO UWB time reversal systems," in Proceedings of the IEEE International Conference on Ultra-Wideband (ICUWB '09), pp. 295-299, September 2009.

[27] T. K. Nguyen, H. Nguyen, F. Zheng, and T. Kaiser, "Spatial correlation in SM-MIMO-UWB systems using a pre-equalizer and pre-Rake filter," in Proceedings of the IEEE International Conference on Ultra-Wideband (ICUWB '10), pp. 540-543, September 2010. 
[28] J. Foerster, "Channel modeling sub-committee report final," IEEE, Document IEEE P802.15-02/490r1-SG3a, 2003, http // www.ieee802.org/15/pub/2003/Mar03/02490r1P802-15_SG 3a-Channel-Modeling-Subcommittee-Report-Final.zip.

[29] W. Q. Malik, "Spatial correlation in ultrawideband channels," IEEE Transactions on Wireless Communications, vol. 7, no. 2, pp. 604-610, 2008.

[30] T. Kaiser, F. Zheng, and E. Dimitrov, "An overview of ultrawide-band systems with MIMO," Proceedings of the IEEE, vol. 97, no. 2, pp. 285-312, 2009.

[31] A. Paulraj, R. Nabar, and D. Gore, Introduction to Space-Time Wireless Communications, Cambridge University Press, 2003.

[32] S. L. Loyka, "Channel capacity of MIMO architecture using the exponential correlation matrix," IEEE Communications Letters, vol. 5, no. 9, pp. 369-371, 2001.

[33] A. V.Zelst and J. Hammerschmidt, "A single coefficient spatial correlation model for Multiple-input Multiple-output radio channels," in 27th General Assembly of the International Union of Radio Science, 2002.

[34] H. Nguyen, Z. Zhao, F. Zheng, and T. Kaiser, "Preequalizer design for spatial multiplexing SIMO-UWB TR systems," IEEE Transactions on Vehicular Technology, vol. 59, no. 8, pp. 3798$3805,2010$.

[35] R. M. Buehrer, A. Safaai-Jazi, W. Davis, and D. Sweeney, "Ultrawideband propagation measurements and modeling, final report," in DARPA NETEX Program, Virginia Tech, 2004.

[36] I. Oppermann, M. Hmlinen, and J. Iinatti, UWB Theory and Applications, John Wiley \& Sons, 2004. 

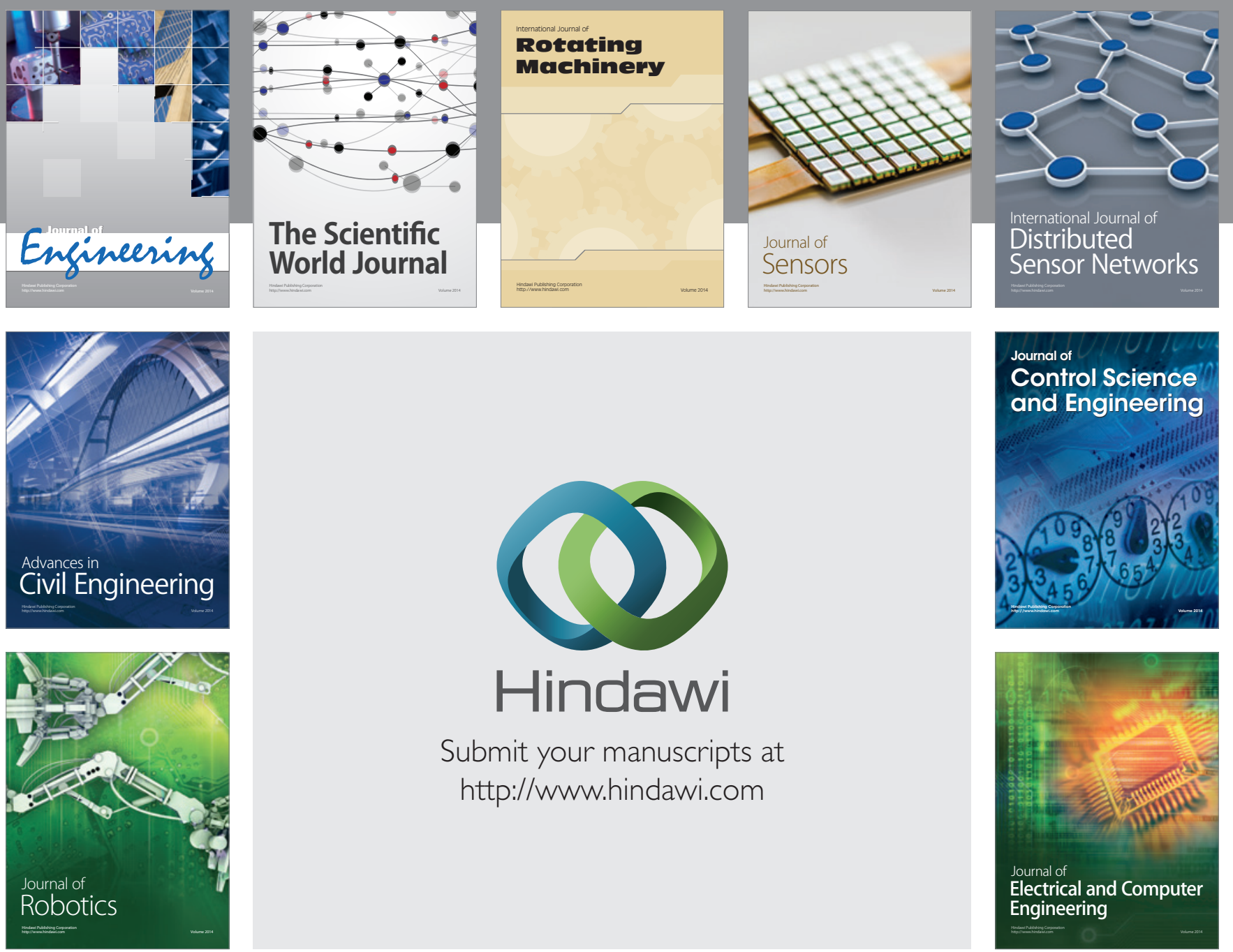

Submit your manuscripts at

http://www.hindawi.com
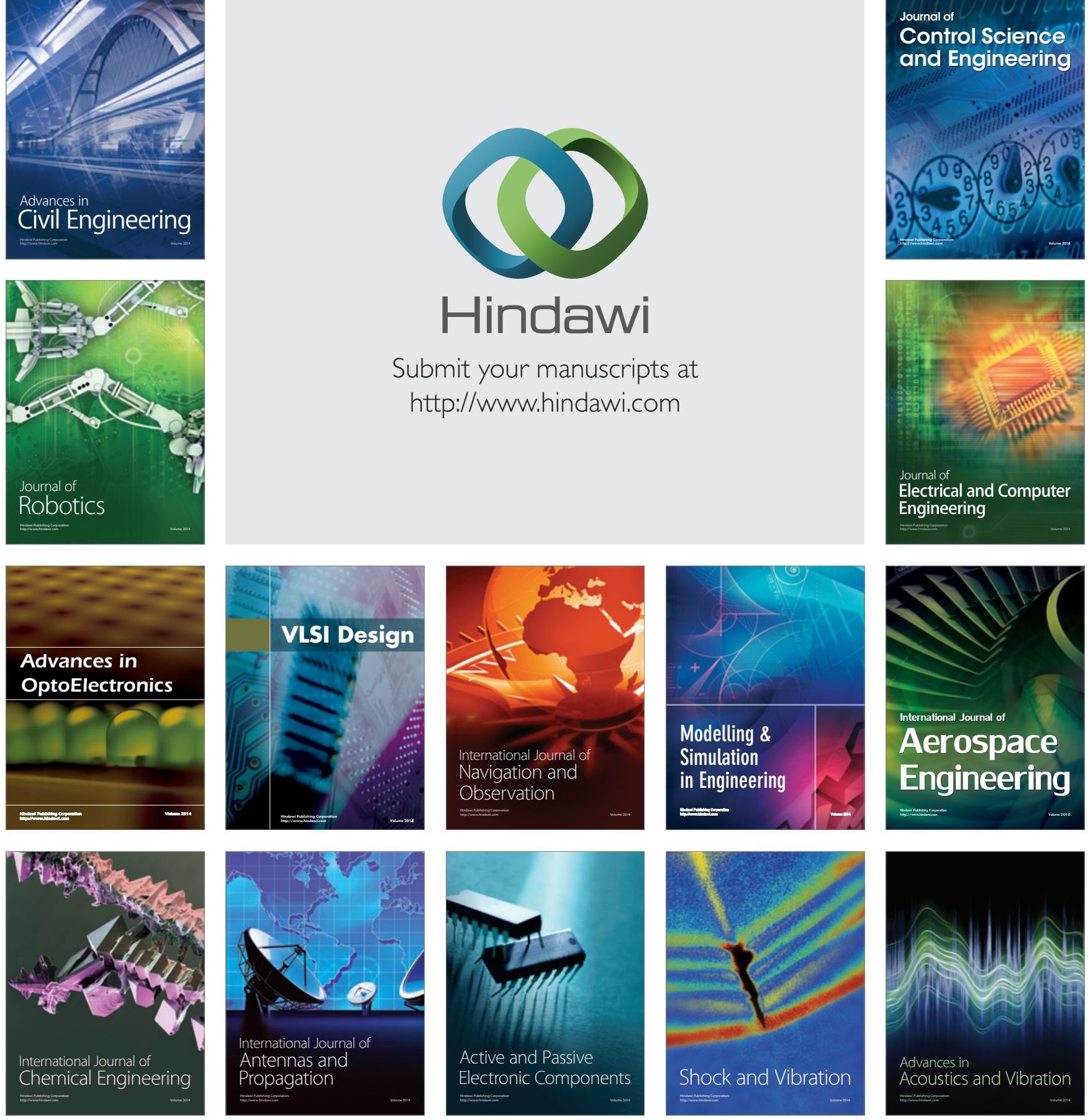